\title{
Otto Hanš
}

Reduzierende zufällige Transformationen

Czechoslovak Mathematical Journal, Vol. 7 (1957), No. 1, 154-158

Persistent URL: http://dml.cz/dmlcz/100238

\section{Terms of use:}

(C) Institute of Mathematics AS CR, 1957

Institute of Mathematics of the Czech Academy of Sciences provides access to digitized documents strictly for personal use. Each copy of any part of this document must contain these Terms of use.

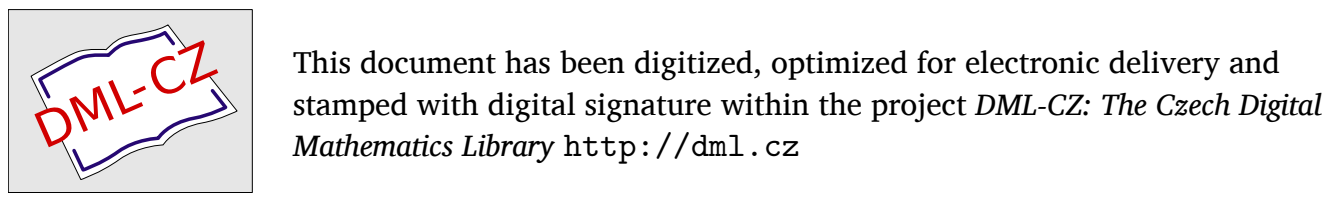




\title{
REDUZIERENDE ZUFÄLLIGE TRANSFORMATIONEN
}

\author{
OTTO HANŠ, Praha.
}

(Eingelangt 14. Feber 1956.)

\begin{abstract}
Es ist oft möglich, die Frage der Existenz und Eindeutigkeit der Lösungen von verschiedenen Gleichungen auf die Anwendung des klassischen Prinzips der reduzierenden Transformationen zurückzuführen. In dieser Arbeit wird gezeigt, dass eine wahrscheinlichkeitstheoretische Verallgemeinerung dieses Prinzips nicht nur bei Existenzund Eindeutigkeitsfragen sondern auch für die Beweise der Messbarkeit der Lösungen von zufälligen Gleichungen benützt werden kann.
\end{abstract}

Eine Transformation $T$ des metrischen Raumes $X \neq \emptyset$ in sich wird reduzierend genannt, wenn eine Konstante $0 \leqq c<1$ existiert, sodass für jedes Paar $x, y \in X$

$$
\varrho(T(x), T(y)) \leqq c \cdot \varrho(x, y),
$$

wobei $\varrho$ eine Metrik in $X$ bedeutet. Es ist wohlbekannt, dass für jede reduzierende Transformation des vollständigen Raumes $X$ in sich genau ein Fixpunkt, d. h. genau ein $x_{0} \in X$ mit der Eigenschaft $T\left(x_{0}\right)=x_{0}$ existiert, der durch sukzessive Approximationen erreicht werden kann.

Um von wahrscheinlichkeitstheoretischen Verallgemeinerungen sprechen zu können, müssen wir zuerst die Begriffe der verallgemeinerten zufälligen Grösse und der zufälligen Transformation einführen. Es sei $(\Omega, \Im)$ ein Ereignisraum, d. h. ein abstrakter Raum $\Omega \neq \emptyset$ und eine $\sigma$-Algebra $\mathfrak{S}$ von Teilmengen von $\Omega$. Eine Abbildung $g$ von $\Omega$ in $X$ heisst verallgemeinerte zufällige Grösse, wenn

$$
\{\{\omega: g(\omega) \in B\}: B \in \mathfrak{B}\} \subset \widetilde{S},
$$

oder, was offenbar dasselbe ist,

$$
\{\{\omega: g(\omega) \in G\}: G \in(\mathfrak{S}\} \subset \mathbb{S},
$$

wobei $\mathfrak{B}$ die $\sigma$-Algebra der Borelschen Teilmengen in $X, \mathrm{~d}$.h. die durch das System (S) aller in Bezug auf die Metrik $\varrho$ abgeschlossenen Teilmengen von $X$ erzeugte $\sigma$-Algebra bedeutet. In ähnlicher Weise heisst eine Abbildung $T$ von $\Omega \times Y$. in $X$, wobei $Y \neq \emptyset$ einen abstrakten Raum bedeutet, zufällige 
Transformation, falls für jedes $z \in Y$ die Abbildung $T(., z)$ von $\Omega$ in $X$ eine verallgemeinerte zufällige Grösse ist.

Wenn z. B. $\Omega$ den Raum der für $0 \leqq t \leqq 1,0 \leqq s \leqq 1$ definierten stetigen Funktionen $\omega, R$ den Raum der reellen Zahlen mit der Metrik $\varrho(x, y)=|x-y|$, $X=Y=C$ den Raum aller reeller im abgeschlossenen Interval $\langle 0,1\rangle$ stetigen Funktionen mit der Metrik $\varrho(x, y)=\max _{0 \leq t \leq 1}|x(t)-y(t)|$ und $\mathfrak{S}$ die durch das System $\{\{\omega: \omega(t, s) \leqq r\}: 0 \leqq t \leqq 1,0 \leqq s \leqq 1, r \in R\}$ erzeugte $\sigma$-Algebra bedeuten, so ist die Transformation $y(t)=\int_{0} \omega(t, s) x(s) \mathrm{d} s$ eine zufällige Transformation.

Definiert man für jedes $G \in(S)$ die Abbildung $f_{a}$ von $X$ in $R$ als $f_{\theta}(x)=$ $=\varrho(x, G)=\min _{z_{\epsilon} G} \varrho(x, z)$, so gilt folgendes

Kriterium. Es sei $(\Omega,(\mathfrak{S )})$ ein Ereignisraum und $X$ ein metrischer Raum. Die Abbildung $g$ von $\Omega$ in $X$ ist dann und nur dann eine verallgemeinerte zufällige Grösse, wenn für jedes $G \epsilon$ (S) die Abbildung $f_{\theta}(g)$ von $\Omega$ in $R$ eine gewöhnliche zufällige Grösse ist.

Beweis. Aus der Stetigkeit der Abbildung $f_{a}$ von $X$ in $R$ folgt sofort, dass für eine beliebige verallgemeinerte zufällige Grösse $g$ die Abbildung $f_{G}(g)$ für jedes $G \in(\mathfrak{S}$ eine gewöhnliche zufällige Grösse ist. Wenn jetzt

$$
\left\{\left\{\omega: f_{G}(g(\omega)) \leqq r\right\}: G \in(\mathfrak{S )}, r \in R\} \subset \mathbb{S},\right.
$$

so folgt die umgekehrte Behauptung aus der Identität

$$
\{\{\omega: g(\omega) \epsilon G\}: G \in \mathbb{B S}\}=\left\{\left\{\omega: f_{G}(g(\omega)) \leqq 0\right\}: G \in \mathbb{G}\right\} .
$$

Für unsere weitere Betrachtungen ist folgender Satz nützlich:

Satz 1. Es sei $\left(\Omega, S_{)}\right)$ein Ereignisraum, $X$ ein metrischer Raum und $g_{1}, g_{2}, \ldots$ eine Folge von verallgemeinerten zufälligen Grössen, die für jedes $\omega \epsilon \Omega$ gegen $g(\omega) \epsilon X$ konvergiert.

Dann ist die Abbildung $g$ von $\Omega$ in $X$ eine verallgemeinerte zufällige Grösse.

Beweis. Der Satz folgt sofort aus unserem Kriterium und aus der Gültigkeit dieses Satzes für $X=R$.

Wir wollen sagen, dass die zufällige Transformation $T$ von $\Omega \times X$ in $X$ reduzierend ist, falls für jedes $\omega \in \Omega T(\omega,$.$) eine reduzierende Transformation$ ist. Wenn der ,,reduzierende Koeffizient" unabhängig von $\omega$ ist, so werden wir von einer gleichmässig reduzierenden zufälligen Transformation sprechen.

Durch Spezialisierung des Raumes $X$ und des Typus der reduzierenden zufälligen Transformation $T$ kann man verschiedene zufällige Gleichungen lösen. Im Falle $X=R$ und $X=C$ untersuchte zufällige Gleichungen A. ŠpačEx in [1], der auch in unserer Formulierung folgendermassen lautenden Satz bewiesen hat: 
Es sei $(\Omega, \subseteq)$ ein Ereignisraum, $X$ ein vollständiger metrischer Raum und $T$ eine gleichmässig reduzierende zufällige Transformation von $\Omega \times X$ in $X$, sodass folgende Bedingungen erfüllt sind:

(A) in $\Omega$ existiert eine Metrik $\delta$, sodass $\Omega$ in Bezug auf diese Metrik separabel ist;

(B) $\subseteq$ enthält alle sphärische Umgebungen in $\Omega$;

(C) für jedes feste $x \in X$ ist die Abbildung $T(., x)$ von $\Omega$ in $X$ stetig.

Dann ist die Abbildung $\Phi$, die jedem $\omega \in \Omega$ den Fixpunkt der Transformation $T(\omega,$.$) zuordnet, eine verallgemeinerte zufällige Grösse.$

Ein ähnliches für Anwendungen nützliches Resultat in dieser Richtung ist der

Satz 2. Es sei $(\Omega$, S) ein Ereignisraum, $X$ ein vollständiger separabler metrischer Raum und T eine reduzierende zufällige Transformation von $\Omega \times X$ in $X$.

Dann existiert genau eine verallgemeinerte zufällige Grösse $\Phi$, die jedem $\omega \epsilon \Omega$ den Fixpunkt der Transformation $T(\omega,$.$) zuordnet.$

Beweis. Es sei $\left\{x_{1}, x_{2}, \ldots\right\}$ eine abzählbare dichte Menge in $X$ und

$$
A_{i n}=\left\{x: \varrho\left(x, x_{i}\right) \leq \frac{1}{n}\right\}-\bigcup_{j=1}^{i} A_{j n} \quad i=1,2, \ldots
$$

Für $x \in A_{i n}$ setzen wir $T_{n}(., x)=T\left(., x_{i}\right)$, sodass

$$
\left\{(\omega, x): T_{n}(\omega, x) \in B\right\}=\mathbf{U}_{j=1}^{\infty}\left\{\omega: T\left(\omega, x_{i}\right) \in B\right\} \times A_{i n}
$$

und so nach Satz $1\{\{(\omega, x): T(\omega, x) \epsilon B\}: B \in \mathfrak{B}\} \subset \mathfrak{S} \times \mathfrak{B}$ wobei $\mathfrak{S} \times \mathfrak{B}$ die durch das System $\{E \times B: E \in \mathfrak{S}, B \in \mathfrak{B}\}$ erzeugte $\sigma$-Algebra bedeutet. Deswegen ist für jede verallgemeinerte zufällige Grösse $g$ auch die Abbildung $h$ von $\Omega$ in $X$, die für jedes $\omega \epsilon \Omega$ durch $h(\omega)=T(\omega, g(\omega))$ definiert ist, eine verallgemeinerte zufällige Grösse. Es sei $g_{1}$ eine beliebige verallgemeinerte zufällige Grösse und setzen wir für jedes $\omega \epsilon \Omega: g_{n+1}(\omega)=T\left(\omega, g_{n}(\omega)\right), n=1,2, \ldots$ Dann ist $g_{1}, g_{2}, \ldots$ eine Folge von verallgemeinerten zufälligen Grössen, die für jedes $\omega \in \Omega$ nach dem klassischen Prinzip der reduzierenden Transformationen gegen einen einzigen Fixpunkt $\Phi(\omega)$ der Transformation $T(\omega,$.$) kon-$ vergiert. Da nach Satz 1 ist die Abbildung $\Phi$ von $\Omega$ in $X$ eine verallgemeinerte zufällige Grösse, w. z. b. w.

Aus dem vorhergehenden Beweis folgt sofort, dass der Satz 2 folgendermassen ergänzt werden kann:

Satz 3. Es sei $(\Omega$, S) ein Ereignisraum, $X$ ein vollständiger separabler metrischer Raum und $T$ eine reduzierende zufällige Transformation von $\Omega \times X$ in $X$. Es sei $g_{1}$ eine beliebige verallgemeinerte zufällige Grösse und man setze für jedes $\omega \in \Omega$ und für $n=1,2, \ldots: g_{n+1}(\omega)=T\left(\omega, g_{n}(\omega)\right)$. 
Dann ist $g_{1}, g_{2}, \ldots$ eine Folge von verallgemeinerten zufälligen Grössen, die gegen die verallgemeinerte zufällige Fixpunktgrösse $\Phi$ der zufälligen Transformation $T$ konvergiert.

Es sei noch bemerkt, dass zum Beweis der beiden letzten Sätze weder die Bedingungen (A) bis (C) noch die Gleichmässigkeit der Reduktion der zufälligen Transformation $T$ gebraucht wurde. Dagegen wird die Separabilität des Raumes $X$ hinzugenommen, was aber vom Gesichtspunkt der Anwendungen auf zufällige Gleichungen keine wesentliche Beschränkung bedeutet.

Jetzt können wir schon folgenden Satz formulieren:

Satz 4. Es sei $\Omega$ der Raum der für $0 \leqq t \leqq 1,0 \leqq s \leqq 1$ definierten, stetigen und durch die Konstante $\alpha>0$ beschränkten Funktionen $\omega, x_{0} \in X=C$, $\{\{\omega: \omega(t, s) \leqq r\}: 0 \leqq t \leqq 1,0 \leqq s \leqq 1, r \in R\} \subset \Im$ und $|\beta|<1 / \alpha$.

Dann ist die Abbildung $\Phi$, die jedem $\omega \in \Omega$ die Lösung der zufälligen Fredholmschen Integralgleichung

$$
x(t)=x_{0}(t)+\beta \int_{0}^{1} \omega(t, s) x(s) \mathrm{d} s
$$

zuordnel, eine verallgemeinerte zufällige Grösse, die durch sukzessive A pproximationen erreicht werden kann.

Beweis. Der Satz folgt sofort aus dem Beispiel der zufälligen Transformation und aus unseren allgemeinen Sätzen.

Man sieht leicht ein, dass die vorhergehenden Betrachtungen anstatt sicher auch mit Wahrscheinlichkeit 1 durchgeführt werden können.

\section{LITERATUR}

[1] A. Šaček: Zufällige Gleichungen, Czechoslovak Math. J. 5 (80), 1955, 462-466.

$$
\text { Резюме }
$$

\section{СЖАТЫЕ СЛУЧАЙНЫЕ ОТОБРАЖЕНИЯ \\ OTTO ХАНШ (Otto Hanš), Прага. \\ (Поступило в редакцию 14/II 1956 г.)}

Так как принцип сжатых отображений играет важную роль в вопросах существования и единственности решений разных типов уравнений, то вероятностное обобщение этого принципа имеет значение не только для вопросов существования и единственности, но даже для вопросов измеримости решений случайных уравнений. В статье определены понятия обоб- 
щенной случайной величины, случайного отображения и сжатого случайного отображения. Далее доказаны следующие теоремы.

Теорема 1. Пусть ( $\Omega$, S) - пространство событий, $X$ - метрическое пространство и $g_{1}, g_{2}, \ldots$ - последовательность обобщенных случайных величин, сходячаяся для всякого $\omega \in \Omega \kappa$ точке $g(\omega) \epsilon X$.

Тогда отображсние $g$ пространства $\Omega$ в пространство $X$ явяяется обобщенной случайной величиной.

Теорема 2. Пусть $(\Omega$, S) - пространство событий, $X$ - полное сепарабельное метрическое пространство и $T$ - сжатое случайное отображсение пространства $\Omega \times X$ в пространство $X$.

Тогда существует точно одна обобченная случайная величина $\Phi$, которая всякому $T(\omega,$.$) .$ 